\title{
UTILITY OF TRIGLYCERIDE-GLUCOSE INDEX IN PREDICTING GLYCEMIC CONTROL IN TYPE 2 DIABETES MELLITUS
}

\author{
Santosh Timalsina ${ }^{1 *}$, Shishir Mahato ${ }^{2}$, Sandesh Nepal ${ }^{2}$
}

\begin{abstract}
Affiliation
1. Assistant Professor, Department of Biochemistry, Chitwan Medical College, Nepal.

2. Student, Department of Laboratory Medicine, Chitwan Medical College, Nepal.
\end{abstract}

\section{ARTICLE INFO}

Received : 12 December, 2020

Accepted : 14 September, 2021

Published : 04 November, 2021

(C) Authors retain copyright and grant the journal right of first publication with the work simultaneously licensed under Creative Commons Attribution License CC - BY 4.0 that allows others to share the work with an acknowledgment of the work's authorship and initial publication in this journal.

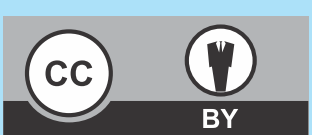

ORA 248

DOI: https://doi.org/10.3126/bjhs.v6i2.40308

\author{
* Corresponding Author \\ Dr. Santosh Timalsina \\ Assistant Professor \\ Department of Biochemistry \\ Chitwan Medical College \\ Email: timalsina.santosh@cmc.edu.np \\ ORCID: https://orcid.org/0000-0003-4425-5614
}

\section{Citation}

Santosh Timalsina, Shishir Mahato, Sandesh Nepal. Utility of Triglyceride- Glucose index in predicting glycemic control in type 2 diabetes mellitus. BJHS 2021;6(2)15. 1444-1448.

\section{ABSTRACT}

\section{Introduction}

Insulin resistance (IR) and glycemic control are two very important aspects to be considered during management of patients with Type 2 Diabetes Mellitus (T2DM). The triglyceride-glucose (TyG) index has been proposed as a simple and inexpensive parameter that correlates well with IR and glycemic control.

\section{Objectives}

To explore the association of TyG index (and other TyG derived indices) with glycated hemoglobin (HbA1c) and evaluate their predictive ability for glycemic control in patients with T2DM.

\section{Methodology}

This cross-sectional study comprised of 160 adult patients diagnosed with T2DM visiting the medical outpatient department of Chitwan Medical College, Bharatpur, Chitwan between July-December 2019. Socio-demographic data and anthropometric measurements were collected. Glycemic control was assessed by HbA1c. TyG index was calculated by the formula: In [fasting TG $(\mathrm{mg} / \mathrm{dl}) \mathrm{x}$ fasting glucose $(\mathrm{mg} / \mathrm{dl}) / 2$ ]. Receiver operating characteristic (ROC) curve analysis was performed to analyze the predictive ability of TyG-index for poor glycemic control.

\section{Result}

One hundred and sixty patients (mean age: $53.6 \pm 10.7$ years, $55.0 \%$ males) were included in the study. Eighty $(50.0 \%)$ had good glycemic control (HbA1c <7.0\%). TyG index, along with TyG-BMI and TyG-WC (other TyG derived indices) were significantly increased in the poor glycemic control group. TyG index had a good predictive ability for poor glycemic control (AUC: $0.803,95 \% \mathrm{Cl}: 0.731-0.874)$. A TyG cutoff $\geq 9.12$ was optimal for predicting poor glycemic control, with $86.1 \%$ sensitivity and $61.5 \%$ specificity.

\section{Conclusion}

TyG index could be a simple and cost-effective screening tool for assessment of glycemic control in patients with T2DM.

\section{KEY WORDS}

Glycemic control, Triglyceride glucose index, Type 2 diabetes mellitus 


\section{INTRODUCTION}

The global prevalence of type 2 diabetes mellitus (T2DM), a common metabolic disorder characterized by hyperglycemia and insulin resistance is ever increasing; and has been regarded as a major public health issue. ${ }^{1}$ In a recent nationwide survey in Nepal, the prevalence of diabetes among adults was $5.8 \%$, and a substantial gap in the diagnosis and treatment was observed. ${ }^{2}$

Glycosylated hemoglobin (HbA1c) is the commonly used assessment tool for glycemic control, which reflects average glycemia over approximately 3 months. It has been shown to have a strong predictive value for diabetes complications. ${ }^{3,4}$ The frequency of $\mathrm{HbA} 1 \mathrm{c}$ testing has been recommended to be guided by the clinical situation, treatment regimen and the clinicians' judgement according to recent guidelines ${ }^{5}$, however frequent testing in low-income countries is still limited by its high cost and inadequate availability of standardized assays. ${ }^{6}$

The triglyceride-glucose (TyG) index is a simple, inexpensive parameter that has been suggested as a surrogate marker for insulin resistance, closely correlating with Homeostasis Model Assessment of Insulin Resistance (HOMA-IR). ${ }^{7}$ Insulin resistance supposedly contributes to poor glycemic control, hypertension, dyslipidemia and accelerated atherosclerosis. ${ }^{8}$ Accordingly, TyG index has been identified as a predictive marker for coronary artery atherosclerosis and nonalcoholic fatty liver disease (NAFLD). ${ }^{9,10}$ Furthermore, several studies have shown good association of TyG index with glycemic control, suggesting its potential role in T2DM patient assessment and management. ${ }^{11,12}$ The aim of this study was to explore the association of TyG index (and other TyG derived indices) with $\mathrm{HbA} 1 \mathrm{c}$ and evaluate their predictive ability for glycemic control in patients with T2DM. We expect TyG index to have a satisfactory ability to discriminate patients with poor glycemic control, suggesting its clinical utility in type 2 DM.

\section{METHODOLOGY}

This cross-sectional analytical study comprised of 160 adult patients diagnosed with type 2 diabetes mellitus (T2DM) visiting the medical outpatient department of Chitwan Medical College, Bharatpur, Chitwan. The sample size was calculated using the sample size formula for estimating a sample size necessary to estimate an AUROC curve (Source: https://sample-size.net/sample-size-ci-for-auroc/). Based on a previous study, ${ }^{12}$ using an estimated AUROC $(\theta)=0.83$, estimated proportion of diabetics having poor glycemic control $(P)=0.46$, width of confidence interval $(0.13)$ at $95 \%$ confidence level, the calculated sample size was 163 . Convenience sampling method was used.

This study was conducted from July -December 2019. Patients under thyroid medications or steroid therapy (that could alter blood glucose), pregnant women, patients with type 1 diabetes mellitus and other co-existing serious illness or inflammation were excluded from the study. Research participants who had highly elevated serum triglyceride (TG) (>500 mg/dl) or had taken medications lowering primarily serum TG (e.g. fenofibrate) were also excluded. Ethical approval was obtained from Chitwan Medical
College-Institutional Review Committee [Ref No. CMCIRC/076/077-039].

The diagnosis of T2DM was made based on the wellestablished American Diabetes Association (ADA) criteria. ${ }^{13}$ After explaining about the study and receiving written consent, socio-demographic data and clinical data including the anthropometric measurements [weight, height, waist circumference (WC)] were collected from the research participants, following standard protocol on day 1 . On the nextmorning (day 2), about $5 \mathrm{ml}$ of blood was drawn from the patients in the fasting state (a minimum 8 hours of fasting) by venipuncture using aseptic technique in two vacutainers: one for serum analysis (vacutainer with clot activator) and the other for $\mathrm{HbA1c}$ measurement in whole blood (vacutainer with the antocoagulant EDTA). The biochemical parameters included fasting blood glucose (FBG), lipid profile [Total cholesterol (TC), Triglyceride (TG), Low-density lipoprotein-cholesterol (LDL-C), High-density lipoprotein-cholesterol (HDL-c)] and glycated hemoglobin (HbA1c). The measurement of glucose and lipid parameters was done by standard colorimetric assays using DIMENSION Clinical Chemistry System, SIEMENS. HbA1c was measured by lon-exchange High Performance Liquid Chromatography (HPLC) method.

The patients were categorized into two groups of glycemic control based on HbA1c levels; < $7.0 \%$ as "good glycemic control" and $\geq 7.0 \%$ as "poor glycemic control". The selection of these cutoff values was based on earlier studies. ${ }^{14}$ The categorization of patients into different obesity statuses was based on WHO classification based on Body Mass Index (BMI). ${ }^{15}$

TyG indices were calculated according to established formulae.,16

TyG index = In [fasting TG (mg/dl) x fasting glucose (mg/dl)/2]

TyG-WC $=$ TyG index $*$ WC

TyG-BMI = TyG index * BMI

Statistical Package for the Social Sciences (SPSS) ver. 20 was used to analyze the data. Normality in data distribution in the variables was assessed by Shapiro Wilk test. Continuous variables were expressed as mean $\pm \mathrm{SD}$ or median $(\mathrm{Q} 1-\mathrm{Q} 3)$ depending upon their distribution, whereas categorical variables were expressed as frequency (\%). The comparison of different continuous variables between good and poor glycemic control groups was done by Independent sample ttest or Mann-Whitney $U$ test as appropriate. Chi-squared test was used to analyze the difference in proportion between groups, for categorical variables. Spearman's rank correlation coefficient (rho) was used to explore correlation between variables. Receiver operating characteristic (ROC) curve analysis was performed to analyze the predictive ability of TyG-index and TyG-WC for poor glycemic control. The optimal cut-off was derived based on the value that had maximal sensitivity and specificity. $\mathrm{P}<0.05$ was considered to be statistically significant.

\section{RESULTS}

A total of 160 patients (mean age: $53.6 \pm 10.7$ years) were included in the study. Eighty-eight (55.0\%) were males. Table 1 describes the baseline characteristics of the study 
population. Almost half of the patients $(73,45.6 \%)$ were overweight. Eighty (50.0\%) had good glycemic control ( $\mathrm{HbA} 1 \mathrm{c}<7.0 \%)$.

\begin{tabular}{|c|c|}
\hline Variable & Summary statistic \\
\hline Age (years), mean \pm SD & $53.6 \pm 10.7$ \\
\hline Gender, n (\%) & \\
\hline Male & $88(55.0)$ \\
\hline Female & $72(45.0)$ \\
\hline Height $(\mathrm{cm})$, mean \pm SD & $155.6 \pm 6.8$ \\
\hline Weight $(\mathrm{kg})$, mean $\pm S D$ & $68.2 \pm 9.8$ \\
\hline Waist circumference $(\mathrm{cm})$, mean $\pm S D$ & $92.4 \pm 10.6$ \\
\hline $\mathrm{BMI}\left(\mathrm{kg} / \mathrm{m}^{2}\right)$, mean $\pm \mathrm{SD}$ & $28.2 \pm 4.4$ \\
\hline Classification based on BMI, $\mathrm{n}(\%)$ & \\
\hline Underweight & $3(1.9)$ \\
\hline Normal weight & $34(21.2)$ \\
\hline Overweight & $73(45.6)$ \\
\hline Obese & $50(31.3)$ \\
\hline Duration of DM (years), median (IQR) & $3.0(1.4-5.0)$ \\
\hline $\mathrm{FBG}(\mathrm{mg} / \mathrm{dl})$, mean $\pm \mathrm{SD}$ & $164.2 \pm 89.9$ \\
\hline $\mathrm{HbA1c}(\%)$, mean $\pm \mathrm{SD}$ & $7.3 \pm 1.0$ \\
\hline Glycemic control (based on $\mathrm{HbA} 1 \mathrm{c}$ ), $\mathrm{n}(\%)$ & \\
\hline Good control & $80(50.0)$ \\
\hline Poor control & $80(50.0)$ \\
\hline TG (mg/dl), median (IQR) & $154.5(118.0-214.5)$ \\
\hline $\mathrm{TC}(\mathrm{mg} / \mathrm{dl})$, median (IQR) & $183.2(150.0-209.7)$ \\
\hline $\mathrm{HDL}(\mathrm{mg} / \mathrm{dl})$, median (IQR) & $43.0(40.0-47.0)$ \\
\hline LDL (mg/dl), median (IQR) & $100.5(74.8-123.9)$ \\
\hline TyG index, mean $\pm S D$ & $9.40 \pm 0.66$ \\
\hline TyG-BMI, mean \pm SD & $265.34 \pm 49.20$ \\
\hline TyG-WC, mean \pm SD & $867.40 \pm 128.01$ \\
\hline
\end{tabular}

Table 2 illustrates the differences in different clinical and laboratory parameters between patients with poor and good glycemic control. The two groups had similar distribution of age, gender, BMI and duration of DM. As expected, the poor glycemic control group had significantly higher fasting blood glucose (FBG) and glycosylated hemoglobin (HbA1c). TyG index, along with TyG-BMI and TyG-WC were also significantly increased in the poor glycemic control group. [Table 2]

\begin{tabular}{|c|c|c|c|}
\hline Variable & $\begin{array}{c}\text { Poor glycemic } \\
\text { control group } \\
(n=80)\end{array}$ & $\begin{array}{c}\text { Good glycemic } \\
\text { control group } \\
(\mathrm{n}=80)\end{array}$ & P-value \\
\hline Age (years), mean $\pm S D$ & $53.3 \pm 11.5$ & $53.8 \pm 10.0$ & 0.786 \\
\hline $\begin{array}{l}\text { Gender, } \mathrm{n}(\%) \\
\text { Male } \\
\text { Female }\end{array}$ & $\begin{array}{l}41(51.2) \\
39(48.8)\end{array}$ & $\begin{array}{l}47(58.8) \\
33(41.2)\end{array}$ & 0.340 \\
\hline BMI $\left(\mathrm{kg} / \mathrm{m}^{2}\right)$, mean \pm SD & $28.31 \pm 3.97$ & $28.16 \pm 4.75$ & 0.842 \\
\hline $\begin{array}{l}\text { Duration of DM (years), } \\
\text { median (IQR) }\end{array}$ & $3.0(1.0-5.0)$ & $3.0(1.9-5.0)$ & 0.744 \\
\hline $\mathrm{FBG}(\mathrm{mg} / \mathrm{dl})$, mean $\pm \mathrm{SD}$ & $206.7 \pm 110.5$ & $121.7 \pm 20.8$ & $<0.001^{*}$ \\
\hline $\mathrm{HbA1c}(\%)$, mean $\pm \mathrm{SD}$ & $8.01 \pm 0.89$ & $6.53 \pm 0.23$ & $<0.001^{*}$ \\
\hline TG $(\mathrm{mg} / \mathrm{dl})$, median (IQR) & $170.5(139.0-253.5)$ & $137.0(110.0-198.0)$ & $0.002^{\#}$ \\
\hline $\mathrm{TC}(\mathrm{mg} / \mathrm{dl})$, median (IQR) & $184.0(150.0-206.5)$ & $181.7(149.8-217.0)$ & 0.929 \\
\hline $\mathrm{HDL}(\mathrm{mg} / \mathrm{dl})$, median (IQR) & $43.0(40.0-47.0)$ & $43.0(40.0-47.0)$ & 0.743 \\
\hline LDL (mg/dl), median (IQR) & $100.2(67.6-119.8)$ & $102.6(75.1-137.2)$ & 0.293 \\
\hline TyG index, mean $\pm S D$ & $9.73 \pm 0.58$ & $9.07 \pm 0.56$ & $<0.001^{*}$ \\
\hline TyG-BMI, mean \pm SD & $275.21 \pm 45.10$ & $256.22 \pm 51.31$ & $0.018^{\#}$ \\
\hline TyG-WC, mean \pm SD & $902.50 \pm 121.59$ & $834.98 \pm 125.92$ & $<0.001^{*}$ \\
\hline
\end{tabular}

${ }^{\#} p<0.05, * p<0.001$
TyG index had the best positive correlation with $\mathrm{HbA1c}$, compared to TyG-WC and TyG-BMI (Spearman's rho $=0.65$ vs. 0.36 and 0.33 respectively, $P<0.001)$. No significant association was observed between these indices and other lipid profile parameters.

On ROC curve analysis, TyG index had a much better predictive ability for glycemic control compared to other two indices (AUC: $0.803,95 \% \mathrm{Cl}: 0.731-0.874$ ). TyG-BMI had the least discriminating ability (AUC: $0.644,95 \% \mathrm{Cl}$ : $0.556-0.733)$. Fig 1 depicts the ROC curves for TyG index and TyG-WC. A TyG value $\geq 9.12$ was noted to be optimal cutoff for predicting poor glycemic control, with $86.1 \%$ sensitivity and $61.5 \%$ specificity.

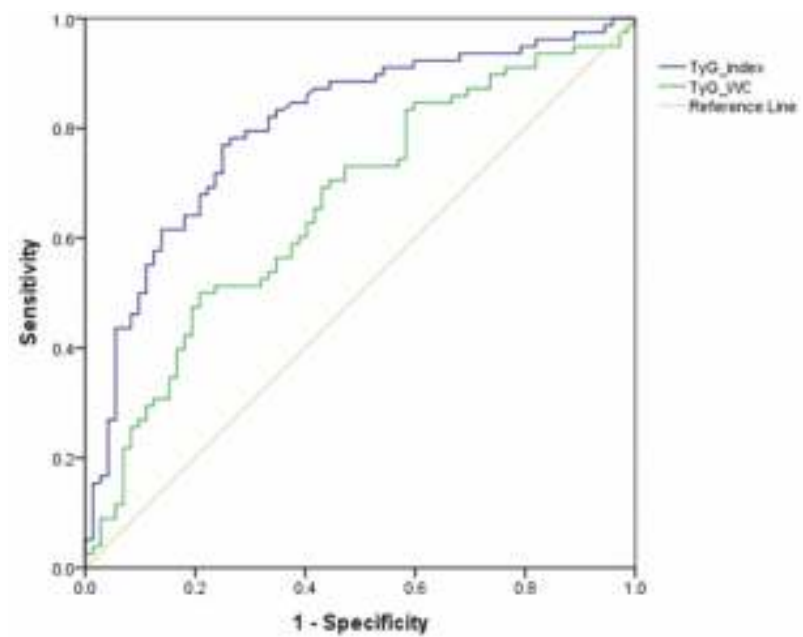

Figure 1: Receiver operating characteristics (ROC) curves for TyG index and TyG-WC and glycemic control

\section{DISCUSSION}

Our study showed that TyG index and other TyG-derived indices (such as TyG-WC and TyG-BMI) are significantly associated with glycemic control in adult patients with type 2 diabetes mellitus. TyG index, in particular showeda good discriminant ability for good glycemic control. The indices had poor correlation with other lipid profile parameters, however.

The product of TG and FBG (TyG index) has been shown to be a surrogate marker for estimating insulin resistance (IR) in healthy subjects, with a high degree of correlation with the commonly used HOMA-IR index and the gold standard euglycemichyperinsulinemic clamp test. ${ }^{7,17}$ IR basically involves two phenomena: reduced sensitivity of the muscle and adipose tissue towards insulin and reduced ability of the liver to suppress hepatic glucose production and output. ${ }^{18}$ Because there is impairment in the oxidation and utilization of fatty acids (FAs) in IR, the resultant increase in the flux of free FAs from adipose to non-adipose tissue amplifies the fundamental metabolic derangements, characteristic of IR. ${ }^{19}$

TyG index has been reported to be a better and efficient marker than other indices such as Visceral Adiposity Index (VAI), lipid ratios and Lipid Accumulation Product (LAP) in early identification of IR. ${ }^{20}$ Similarly, BMI and WC are simple, non-invasive anthropometric parameters that are adopted as indicators of obesity. As obesity and IR are closely related, 
combination of these with TyG index could provide additional value in identifying IR, as has been shown in one of the studies. ${ }^{16}$ Moreover, TyG index has been proposed as a simple biochemical marker that could identify individuals at high risk of developing diabetes in a large cohort study. ${ }^{21}$

In our study, TyG index was significantly and positively associated with $\mathrm{HbA} 1 \mathrm{C}$ in patients with T2DM, also observed in studies elsewhere. ${ }^{11,12}$ A study by Babic et al. in 113 patients showed elevated TyG index in patients with poor glycemic control and suggested the utility of the index in the overweight and obese group among the diabetic patients. ${ }^{11}$ Similarly, Hameed et al. in 293 patients found that TyG and TyG-derived indices (TyG-WC and TyG-BMI) were significantly increased in the "poor glycemic control" group. Moreover, the study also reported that TyG index had the largest AUC (0.833) for prediction of poor glycemic control, as observed in our study (AUC $=0.803) .{ }^{12}$

The dyslipidemia present in T2DM patients is characterized by elevated triglycerides, low HDL-c and predominance of small-dense LDL particles. It is increasingly being recognized that these lipid changes are not only the consequences of the impaired glucose metabolism, but also cause them. ${ }^{22}$ It is hypothesized that elevated levels of free fatty acids (FFAs) in these patients, a consequence of elevated TG, induce insulin resistance and beta-cell dysfunction by a) disrupting or modulating the cascade linking insulin receptors with glucose transporters (GLUTs) and b) inducing subclinical inflammation acting synergistically with pro-inflammatory adipokines released by adipose tissue. ${ }^{23,24}$

It has been shown that comprehensive biomarker testing of IR (and beta cell function) has greater sensitivity to detect diabetes risk and is associated with improved glycemic control in clinical practice. ${ }^{25} \mathrm{HbA} 1 \mathrm{c}$ is the most commonly used and established method for assessment of glycemic control, however the test is expensive, needs to be highly standardized and might be unavailable in many hospital laboratories of low-income countries such as ours. TyG index, a reliable surrogate marker for IR, could be a simple and low-cost alternative for screening for insulin resistance, glycemic control status, and effectiveness of the treatment regimen during patient follow up.

\section{CONCLUSION}

TyG index could be a simple and cost-effective screening tool for assessment of insulin resistance and glycemic control in type 2 diabetic patients, particularly in primary health care setting or when $\mathrm{HbA} 1 \mathrm{c}$ measurement might not be financially/logistically feasible.

\section{RECOMMENDATIONS}

Further prospective researches are needed exploring the potential clinical utility of TyG index in terms of disease progression and effectiveness of diabetic treatment regimen. The reliability of the TyG index has to be confirmed by studies involving a large group of type 2 DM patients.

\section{LIMITATIONS OF THE STUDY}

The major limitations of our study are small sample size and single-center study design. Because of the cross-sectional nature of the study, cause-effect relationships could not be deduced (such as between TyG index and glycemic control). Some of the clinical information, such as use of insulin/oral hypoglycemic agents, family history of diabetes were missing.

\section{ACKNOWLEDGEMENTS}

The author would like to acknowledge staffs from Department of Laboratory Medicine and Medical OPD of Chitwan Medical College for support in data collection and retrieval.

\section{CONFLICT OF INTEREST}

None

\section{FINANCIAL DISCLOSURE}

None

\section{REFERENCES}

1. Global, regional, and national incidence, prevalence, and years lived with disability for 328 diseases and injuries for 195 countries, 19902016: a systematic analysis for the Global Burden of Disease Study 2016. Lancet. 2017;390(10100):1211-59.DOI: https://doi.org/ 10.1016/S0140-6736(17)32154-2

2. Dhimal M BB, Bhattarai S, Dixit LP, Hyder MKA, Agrawal N, et al. Report of Non Communicable Disease Risk Factors STEPS Survey Nepal 2019. Nepal Health Research Council; 2020.Available from: https://www.who.int/docs/default-source/nepal-documents/ ncds/ncd-steps-survey-2019-compressed.pdf?sfvrsn=807bc4c6_2

3. Laiteerapong N, Ham SA, Gao Y, Moffet HH, Liu JY, Huang ES, et al. The Legacy Effect in Type 2 Diabetes: Impact of Early Glycemic Control on Future Complications (The Diabetes \& Aging Study). Diabetes Care. 2019;42(3):416-26.PMID: 30104301

4. Stratton IM, Adler Al, Neil HA, Matthews DR, Manley SE, Cull CA, et al. Association of glycaemia with macrovascular and microvascular complications of type 2 diabetes (UKPDS 35): prospective observational study. Bmj. 2000;321(7258):405-12.PMID: 10938048

5. Introduction: Standards of Medical Care in Diabetes-2020. Diabetes Care.2020;43(Suppl 1):S1-s2.DOI: https://doi.org/10.2337/dc20-Sint

6. Gomez-Perez FJ, Aguilar-Salinas CA, Almeda-Valdes P, Cuevas-Ramos D, Lerman Garber I, Rull JA. HbA1c for the Diagnosis of Diabetes Mellitus in a Developing Country. A Position Article. Archives of Medical Research. 2010;41(4):302-8.PMID: 20637376

7. Simental-Mendía LE, Rodríguez-Morán M, Guerrero-Romero F. The product of fasting glucose and triglycerides as surrogate for identifying insulin resistance in apparently healthy subjects. Metab Syndr Relat Disord. 2008;6(4):299-304.PMID: 19067533

8. Bonora E, Formentini G, Calcaterra F, Lombardi S, Marini F, Zenari L, et al. HOMA-estimated insulin resistance is an independent predictor of cardiovascular disease in type 2 diabetic subjects: prospective data from the Verona Diabetes Complications Study. Diabetes Care. 2002;25(7):1135-41.PMID: 12087010

9. Lee EY, Yang HK, Lee J, Kang B, Yang Y, Lee SH, et al. Triglyceride glucose index, a marker of insulin resistance, is associated with coronary artery stenosis in asymptomatic subjects with type 2 diabetes. Lipids Health Dis. 2016;15(1):155.PMID: 27633375 
10. Zhang S, Du T, Zhang J, Lu H, Lin X, Xie J, et al. The triglyceride and glucose index (TyG) is an effective biomarker to identify nonalcoholic fatty liver disease. Lipids Health Dis. 2017;16(1):15.PMID: 28103934

11. Babic N, Valjevac A, Zaciragic A, Avdagic N, Zukic S, Hasic S. The Triglyceride/HDL Ratio and Triglyceride Glucose Index as Predictors of Glycemic Control in Patients with Diabetes Mellitus Type 2. Med Arch. 2019;73(3):163-8.PMID: 31404127

12. Hameed EK. TyG index a promising biomarker for glycemic control in type 2 Diabetes Mellitus. Diabetes Metab Syndr. 2019;13(1):5603.PMID: 30641766

13. American Diabetes Association. 2. Classification and Diagnosis of Diabetes: Standards of Medical Care in Diabetes-2020. Diabetes Care. 2020 Jan;43(Suppl 1):S14-S31.PMID: 31862745

14. Qaseem A, Wilt TJ, Kansagara D, Horwitch C, Barry MJ, Forciea MA. Hemoglobin A1c Targets for Glycemic Control With Pharmacologic Therapy for Nonpregnant Adults With Type 2 Diabetes Mellitus: A Guidance Statement Update From the American College of Physicians. Ann Intern Med. 2018;168(8):569-76.PMID: 29507945

15. Appropriate body-mass index for Asian populations and its implications for policy and intervention strategies. Lancet. 2004;363(9403):157-63.DOI: https://doi.org/10.1016/S01406736(03)15268-3

16. Er LK, Wu S, Chou HH, Hsu LA, Teng MS, Sun YC, et al. Triglyceride Glucose-Body Mass Index Is a Simple and Clinically Useful Surrogate Marker for Insulin Resistance in Nondiabetic Individuals. PLoS One. 2016;11(3):e0149731.PMID: 26930652

17. Guerrero-Romero F, Simental-Mendía LE, González-Ortiz M, Martínez-Abundis E, Ramos-Zavala MG, Hernández-González SO, et al. The product of triglycerides and glucose, a simple measure of insulin sensitivity. Comparison with the euglycemichyperinsulinemic clamp. J Clin Endocrinol Metab. 2010;95(7):334751.PMID: 20484475
18. Matthaei S, Stumvoll M, Kellerer M, Häring HU. Pathophysiology and pharmacological treatment of insulin resistance. Endocr Rev. 2000;21(6):585-618.PMID: 11133066

19. Lewis GF, Carpentier A, Adeli K, Giacca A. Disordered fat storage and mobilization in the pathogenesis of insulin resistance and type 2 diabetes. Endocr Rev. 2002;23(2):201-29.PMID: 11943743

20. Du T, Yuan G, Zhang M, Zhou X, Sun X, Yu X. Clinical usefulness of lipid ratios, visceral adiposity indicators, and the triglycerides and glucose index as risk markers of insulin resistance. Cardiovasc Diabetol. 2014;13:146.PMID: 25326814

21. Lee SH, Kwon HS, Park YM, Ha HS, Jeong SH, Yang HK, et al. Predicting the development of diabetes using the product of triglycerides and glucose: the Chungju Metabolic Disease Cohort (CMC) study. PLoS One. 2014;9(2):e90430.PMID: 24587359

22. Parhofer KG. Interaction between Glucose and Lipid Metabolism: More than Diabetic Dyslipidemia. Diabetes Metab J. 2015;39(5):35362.PMID: 26566492

23. Lee $Y$, Hirose $H$, Ohneda $M$, Johnson JH, McGarry JD, Unger RH. Betacell lipotoxicity in the pathogenesis of non-insulin-dependent diabetes mellitus of obese rats: impairment in adipocyte-beta-cell relationships. Proc Natl Acad Sci U S A. 1994;91(23):10878-82.PMID: 7971976

24. Rachek LI. Free fatty acids and skeletal muscle insulin resistance. Prog Mol Biol TransI Sci. 2014;121:267-92.PMID: 24373240

25. Varvel SA, Voros S, Thiselton DL, Pottala JV, Dall T, Warnick GR, et al. Comprehensive biomarker testing of glycemia, insulin resistance, and beta cell function has greater sensitivity to detect diabetes risk than fasting glucose and $\mathrm{HbA} 1 \mathrm{c}$ and is associated with improved glycemic control in clinical practice. J Cardiovasc Transl Res. 2014;7(6):597606.PMID: 25070680 